
acts as a plow or as an engraving tool. The ability of the AFM tip to respond inertially to ultrasonic vibration excited perpendicular to the sample surface and dynamically indent hard samples may facilitate the nanoscale machining of semiconductors or engineering ceramics in a reduced time. Figure 3 demonstrates the machining of nanotrenches and holes on a silicon sample in the presence of ultrasonic vibration. Interestingly, no debris is found in the proximity of lithographed areas. Figure 3 (a) refer to results performed using a cantilever with nominal stiffness comprised between 28-91 Nm${ }^{-1}$ and a diamond-coated tip. Figure 4 (b) refer to results achieved using a cantilever with nominal stiffness $0.11 \mathrm{Nm}^{-1}$ and a $\mathrm{SiN}$ tip; in the absence of ultrasound, it was not possible to scratch the Si surface using such a soft cantilever. In the machining of soft materials, as for instance plastic coatings, the ultrasonic-induced reduction of nanoscale friction may permit eventual finer features and improved surface quality in quasi-static approaches. In [9], an in-plane acoustic wave coupled to the sample support was used to enhance the intermittent force exerted by the tip in dynamic AFM nanomachining of thin polymer resist films.

In bottom-up approaches, ultrasound may assist in the self-assembly or AFM manipulation of nanostructures [7]. Effects such as sonolubrication and acoustic levitation have been studied at the microscale. These phenomena may facilitate a tip-induced motion of nano-objects. In the manipulation of nanoparticles (NPs) on surfaces with the tip of an AFM cantilever, when ultrasound is excited at a sample surface both tipparticle and particle-surface frictional properties change [10]. Moreover, the excitation of NP high-frequency internal vibration modes may also modify the NP dynamic re- sponse, and introduce novel mechanisms of particle motion. Some of the opportunities in ultrasonic-assisted AFM manipulation are illustrated in figure 4, which show images of $\mathrm{Au}$ NPs on a silicon surface. The surface was covered by poly-l-lysine to prevent that the $\mathrm{Au}$ NPs were swept away by the AFM tip. Nevertheless, when scanning in contact mode in the absence of ultrasound, most NPs were inevitably swept away by the tip. Scanning in the same conditions that led to a NP displacement but in the presence of surface ultrasonic vibration, with an appropriate election of the ultrasonic amplitude, the NPs remained undisturbed. In figure 4, two NP were displaced while recording the images, which have been pointed out by blue arrows. A close inspection of the data in figure 4 reveals traces of the UFM and LFM responses from those moving particles while being in motion (see areas enclosed by ellipses and rectangles). The study of the UFM response of a moving NP may allow us to learn about the dynamic mechanisms of NP displacement across surfaces. Consistently with the fact that ultrasound eliminates friction, no frictional contrast is distinguished on the undisturbed $\mathrm{Au}$ NPs in the LFM images. However, from the comparison of the traces of the two moving NPs in the forward and backward LFM scans, friction during the tip-induced NP motion is apparent. Controlled and accurate measurements of lateral and ultrasonic forces exerted by individual NPs when in motion under tip ultrasonic actuation may bring about a wealth of information about the dissipated energy, ultrasonic lubrication effects, NP dynamics, etc.

Eventually, it should be pointed out that the sensitivity of ultrasonic-AFM to subsurface features makes feasible to monitor subsurface

\section{The New BioMAT ${ }^{\mathrm{TM}}$}

\section{Workstation AFM.}

\section{Perfect Imaging}

\section{Results for}

\section{Opaque Samples.}

\author{
The BioMAT ${ }^{\text {TM }}$ Workstation combines upright optical \\ microscopy with AFM and opens up a wide range of \\ applications for the study of non-transparent specimens. \\ Even in liquids.
}

www.jpk.com

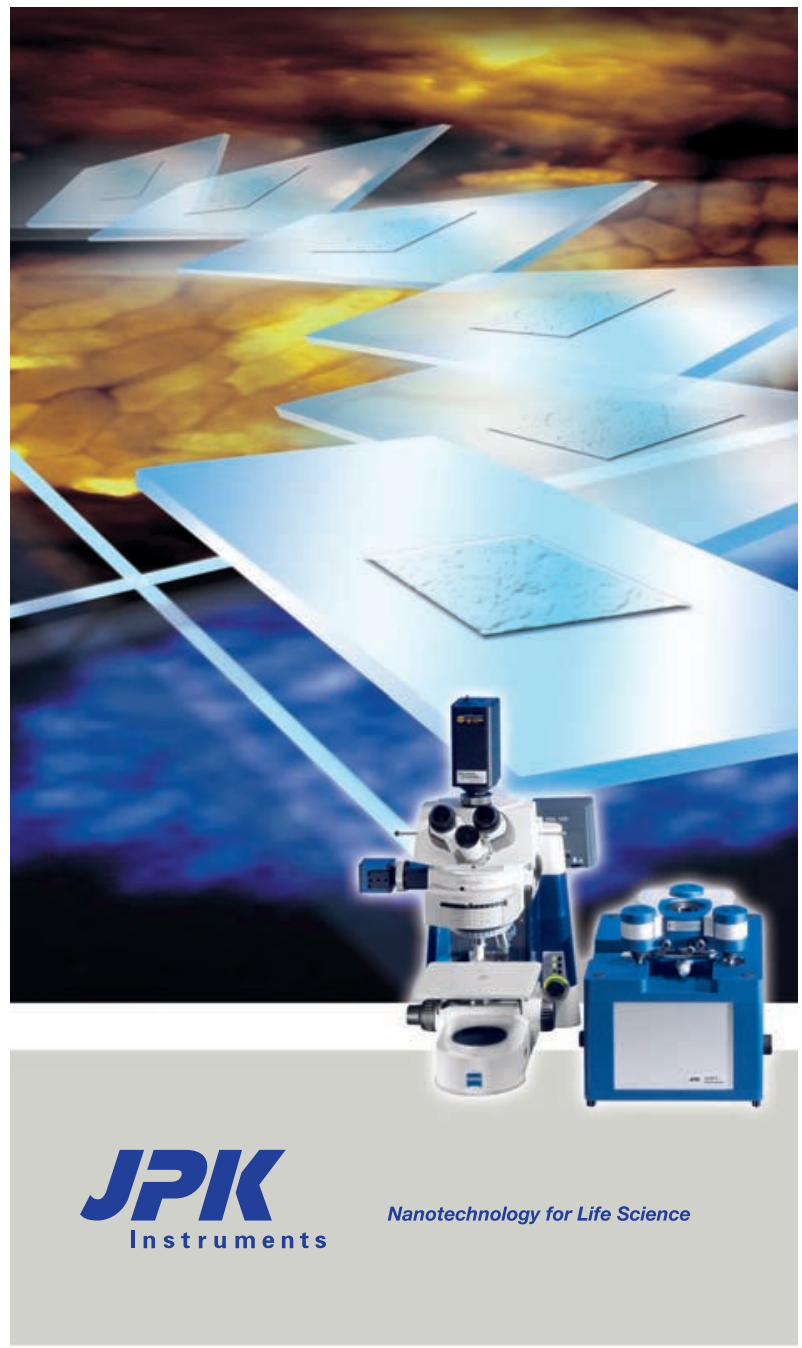




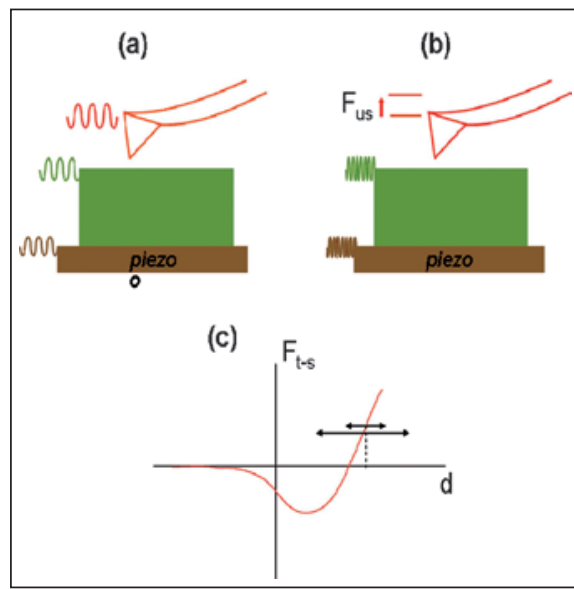

Fig. 1: Detection of surface vibration with the tip of an AFM cantilever. (a) At low frequencies, the tip follows the surface vibration. (b) In the highfrequency regime, for sufficiently high vibration amplitudes, tip experiences an ultrasonic force $F_{u s}$. (c) Tip-sample force $F_{t-s}$ versus tip-sample distance $\mathrm{d}$ curve.

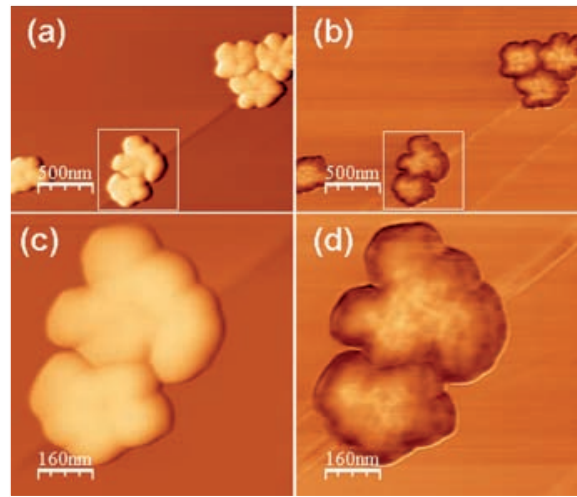

Fig. 2: Sb NPs on HOPG. (a, b) Simultaneously recorded contact-mode AFM topography (a) and UFM image (b). (c, d) Simultaneously recorded high-resolution images from the areas enclosed by white squares in (a), (b). Set point: $1 \mathrm{nN}$; Kc: $0.11 \mathrm{Nm}^{-1}$; UFM parameters: $2.2 \mathrm{MHz}, 8 \mathrm{Vpp}$. Contrast in UFM indicates stiffness variations.
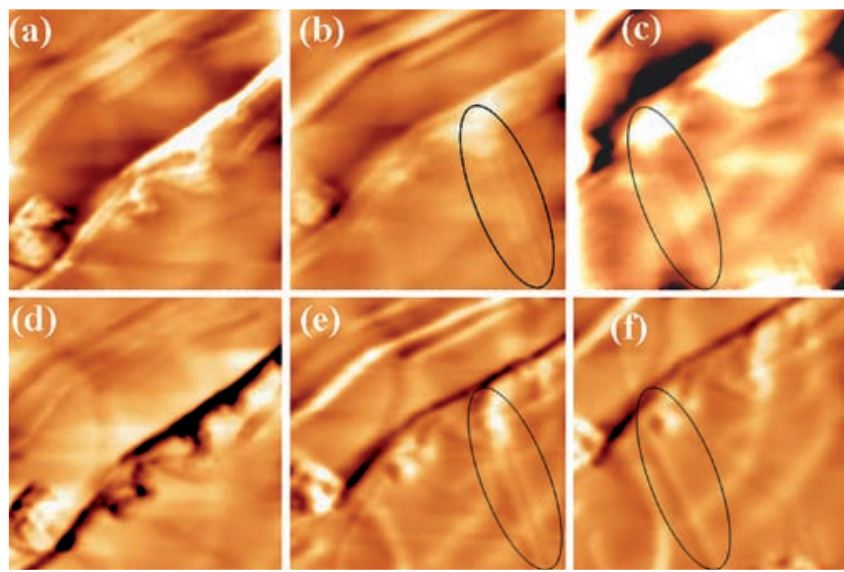

Fig. 5: Lateral displacement of a subsurface dislocation in HOPG by ultrasonic tip actuation (a) Topography of the HOPG surface in AFM contact mode: $(700 \times 700)$ $\mathrm{nm} ; \mathrm{Fo}=105 \mathrm{nN}$. $(\mathrm{b}, \mathrm{c})$ Ultrasonic-AFM images recorded in sequence over nearly the same surface region:

$\mathrm{a}=2.15 \mathrm{MHz}$ (b) $\mathrm{A}=3.5 \mathrm{Vpp}$ (c) $A=2.4$ Vpp. (d-f) LFM forward scan, simultaneously recorded with $(a-b)$ respectively, with the same parameters.

modifications [7]. We have recently demonstrated that actuation with an AFM tip in the presence of ultrasonic vibration can produce stacking changes of extended grapheme layers, and induce permanent displacements of buried dislocations in Highly Oriented Pyrolytic
Graphite (HOPG). This effect is illustrated in figure 5. In the presence of normal surface ultrasonic vibration, both AFM and LFM images reveal subsurface features [1]. Subsurface modification was brought about in this case by scanning in contact mode, with high set-point forces, and high surface ultrasonic excitation amplitudes [7].

\section{Summary}

Ultrasonic AFM techniques provide a means to monitor ultrasonic vibration at the nanoscale, and open up novel opportunities in nanofabrication technologies. The use of ultrasound may improve both down-top and bottom-down approaches in nanofabrication, facilitating the patterning of nanoscale surface features, the manipulation or self-assembly of nanostructures, and possibly the controlled subsurface manipulation of buried nanoobjects.

\section{Acknowledgments}

The author thanks J. J. Martinez and A. Lusvardi for assistance in the UFM lab. The samples of Sb NPs were provided by C. Ritter and U. Schwarz. The Au NPs were provided by M. A. Gonzalez and M.P. Morales. Financial support from the JCCM (Junta de Comunidades de Castilla-La Mancha) under project PBI-05018 is gratefully acknowledged.

\section{References:}

[1] Gnecco, E. and Meyer, E. (Eds.), Fundamentals of Friction and Wear on the nanometer scale, Springer, 2007, 49-71.

[2] Yamanaka K., et al., Appl. Phys. Lett. 64, 178-180 (1994)

[3] Cuberes, M. T., et al., J. Phys. D.: Appl. Phys. $33,2347-2355$ (2000).

[4] Rohrbeck, W. and Chilla, E., Phys. Status Solidi (a) 131, 69-71(1992).

[5] Cuberes, M. T., et al., Ultramicroscopy 107, 1053-1060 (2007).

[6] Cuberes, M. T. and Martínez, J. J., J. of Phys.: Conf. Ser. 62, 224-228 (2007).

[7] Cuberes, M. T., J. of Phys.: Conf. Ser. 61, 219223 (2007).

[8] Dinelli, F., et al., Appl. Phys. Lett. 71, $1177-$ 1179 (1997).

[9] Rubio-Sierra, F. J., et al., Phys. Stat. Sol. (a) 6 , 1481-1486 (2006).

[10] Cuberes, M. T., Proc. of the $30^{\text {th }}$ Annual Meeting of the Adhesion Society, 430-432 (2007).

Contact:

Dr. M. Teresa Cuberes

Engineering School Professor

University of Castilla-La Mancha

Applied Mechanics and Project Engineering

Laboratory of Nanotechnology

Almadén, Spain

Tel.: +34 902204100 ext. 6045

Fax: +34 926264401

teresa.cuberes@uclm.es

www.uclm.es 